\title{
Hyperglycemia-induced Overexpression of PHLPP1 Compromised the Cardioprotective Effect of Ischemic Postconditioning via Modulating the Akt/Mst1 Pathway Signalling.
}

\author{
Yun Qiu \\ Nanjing Medical University \\ Yuming Meng \\ Nanjing Medical University \\ Yajuan Jia \\ Nanjing Medical University \\ Xuemei Lang \\ Nanjing Medical University \\ Hongmei Zhao \\ Nanjing Medical University \\ Lianshu Ding \\ Nanjing Medical University \\ Tingting Wang \\ Huazhong University of Science and Technology Tongji Medical College \\ Hong Sun \\ Nanjing Medical University \\ Sumin Gao ( $\nabla$ gao666332@sina.com ) \\ Nanjing Medical University https://orcid.org/0000-0003-1697-0557
}

\section{Research Article}

Keywords: Ischemic postconditioning, Hypoxic postconditioning, Myocardial ischemia injury, Diabetes, PHLPP1

Posted Date: February 14th, 2022

DOI: https://doi.org/10.21203/rs.3.rs-1299667/v1

License: (c) (1) This work is licensed under a Creative Commons Attribution 4.0 International License. Read Full License 


\section{Abstract}

Purpose Ischemic postconditioning (IPostC) alleviates myocardial ischemia/reperfusion (IR) injury, but the protective effect is lost in diabetes. PH domain leucine-rich repeat protein phosphatase1 (PHLPP1) is able to inactivate Akt. Our previous study found that PHLPP1 expression was upregulated in diabetic heart. We presumed that attenuation of myocardial injury by IPostC might be hindered by PHLPP1 overexpression in diabetic animals.

Methods and results Non-diabetic and diabetic mice were subjected to $45 \mathrm{~min}$ ischemia followed by $2 \mathrm{~h}$ reperfusion with or without IPostC. H9c2 cells were exposed to normal or high glucose and subjected to 4 $\mathrm{h}$ hypoxia followed by $4 \mathrm{~h}$ reoxygenation with or without hypoxic postconditioning (HPostC). IPostC attenuated postischemic infarction, apoptosis, creatine kinase-MB and oxidative stress, accompanied with increased p-Akt as well as decreased PHLPP1 expression and p-Mst1 in non-diabetic but not in diabetic mice. PHLPP1 knockdown or Mst1 inhibitor reduced hypoxia/reoxygenation (HR)-induced cardiomyocyte damage in $\mathrm{H} 9 \mathrm{c} 2$ cells exposed to normal glucose, but the effect was abolished by $\mathrm{PI3K} /$ Akt inhibitor. HPostC attenuated HR-induced cardiomyocyte injury and oxidative stress accompanied with increased p-Akt as well as decreased PHLPP1 expression and p-Mst1 in H9c2 cells exposed to normal glucose but not high glucose. In addition, HPostC in combination with PHLPP1 knockdown or PHLPP1 knockdown alone reduced cell death and oxidative stress in H9c2 cells exposed to high glucose, which was hindered by PI3K/Akt inhibitor.

Conclusion IPostC prevented myocardial IR injury partly through PHLPP1/Akt/Mst1 signalling, and the abnormality of this pathway may be responsible for the loss of IPostC cardioprotection in diabetes.

\section{Introduction}

According to the 2021 report from the American Heart Association, ischemic heart disease remains one of the leading causes of death worldwide, especially among people with diabetes [1]. Restoring the coronary flow to the ischemic heart is a necessary condition to limit cardiac dysfunction and infarct size, but reperfusion of the ischemic area can further aggravate myocardial ischemia/reperfusion (IR) injury [2]. Ischemic conditioning provides protective effect against cardiac IR injury by mainly activating the reperfusion injury salvage kinase (RISK) and survivor activator factor enhancement (SAFE) signaling [3]. Ischemic postconditioning (IPostC) is a more promising approach to cardiac protection than preconditioning due to the difficulty to predict the occurrence of myocardial ischemia in clinical practice. Our previous study showed that remote limb ischemic postconditioning can protect against myocardial IR injury via the activation of JAK/STAT3-mediated Nrf2-antioxidant signaling [4]. However, diabetes severely impedes the development of cardioprotective responses to postconditioning stimuli by disrupting the activation of cardioprotective signaling pathways [5].

The RISK pathway refers to prosurvival kinases that are activated during the reperfusion phase of IR injury to provide cardiac protection [6]. Akt, one of the major kinases in this pathway, is activated by 
postcondtioning during reperfusion to mitigate myocardial injury [7]. The significant decrease of Akt activity in diabetic myocardium is the main cause of insusceptibility to the protective effect of ischemic postconditioning, and the increase of Akt activity can alleviate the IR injury in the diabetic heart [8]. In our previous study, it was also found that Akt activity was significantly reduced in the diabetic heart $[9,10]$. The PH domain rich leucine repeat protein kinase (PHLPP) is an endogenous negative regulator of Akt activity and dephosphorylates it to terminate its downstream signaling to promote tumor apoptosis [11]. The PHLPP family of phosphatases includes two isoforms, PHLPP-1 and PHLPP-2. The study has shown that phosphorylation of Akt increased significantly in PHLPP1 knockout mice, thereby alleviating myocardial injury after IR [12]. Our previous results indicated that the expression of PHLPP1 was increased in the myocardium of diabetic mice [9]. Taken together, these data led us to speculate that PHLPP-1 abundance could be related to the insusceptibility to the protective effect of ischemic postconditioning in diabetic myocardium.

Mammalian sterile 20-like kinase 1 (Mst1) is a core component of Hippo signaling pathway and a widely expressed pro-apoptotic kinase that plays a key role in cell growth and apoptosis [13]. It shows an altered kinase activity depending on the phosphorylation states at Thr183 and Thr387. PHLPP1 dephosphorylates Mst1 on the Thr387 inhibitory site, which increases phosphorylation of Mst1 on the Thr183 site [14].

In response to various stresses, Mst1 is activated in cardiomyocytes through many different kinds of molecular mechanisms, including cytokines and oxidative stress $[15,16]$. Some studies have shown that inhibition of Mst1 reduced myocardial injury as well as improved cardiac function after myocardial infarction [15, 17]. Meanwhile, suppression of Mst1 inhibited mitochondrial fission and myocardial apoptosis, thus preventing the development of diabetic cardiomyopathy $[18,19]$. Although these results have demonstrated that Mst1 regulates downstream targets to promote apoptosis, its roles in ischemic postconditioning cardioprotection remain to be elucidated.

In this study, PI3K/Akt or Mst1 inhibitor and adenovirus encoding siRNA against PHLPP1 were used in in vivo or vitro model. Accordingly, the aims of the present study were to identify whether IPostC protected against myocardial IR injury via modulating the PHLPP1/Akt/Mst1 signalling and the increased level of PHLPP1 is responsible for the impaired responsiveness of the diabetic heart to IPostC.

\section{Materials And Methods}

\subsection{Animals and induction of diabetes}

Male C57BL/6 mice (25 $\pm 2 \mathrm{~g}$, 8 weeks old) purchased from Shanghai SLRC Laboratory Animal Co., Ltd. (Shanghai, China) were used in this study. All animals were housed in temperature-controlled cages with free access to food and water. All animal studies were approved by the Animal Care and Use Committee of Nanjing Medical University, and the animal procedures were carried out in accordance with the "Guide for the Care and Use of Laboratory Animals" published by the US National Institutes of Health. 
Diabetes was induced by continuous intraperitoneal injection of streptozotocin (STZ) $(40 \mathrm{mg} / \mathrm{kg}$; Sigma-

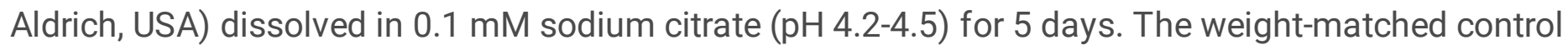
mice received an equivalent volume of vehicle only (sodium citrate). One week after the last injection of STZ, the blood glucose concentrations of all mice were measured and mice with blood glucose levels > $300 \mathrm{mg} / \mathrm{dL}$ were defined as diabetic. At termination (8 weeks after STZ-treatment), mice were weighed and then subjected to myocardial IR as previously described [20].

\subsection{Animal experimental protocol}

The mice were randomly separated into six groups as follows: 1) Sham; 2) IR: nondiabetic mice were subjected to 45 min ischemia followed by 120 min reperfusion; 3) IR+IPostC: nondiabetic C57BL/6 mice received IR and IPostC treatments. IPostC was induced by three cycles of $10 \mathrm{~s}$ of reperfusion and ischemia at the beginning of reperfusion; 4) DM: diabetic C57BL/ 6 mice were threaded the ligature underneath the left anterior descending coronary artery (LAD), but not ligation; 5) DM+IR: diabetic mice underwent IR as previously described; 6) DM+IR+IPostC: diabetic mice underwent IR and IPostC as previously described.

\subsection{Cell protocol}

H9c2 cells obtained from the National Collection of Authenticated Cell Cultures (Shanghai, China) were used for the in vitro model. Cells were cultured in DMEM (Thermo Fisher Scientific, USA) with $5.5 \mathrm{mM}$ (normal glucose) or $25 \mathrm{mM}$ glucose (high glucose) containing 10\% (v/v) FBS (Gibco, USA) under an atmosphere of $5 \% \mathrm{CO} 2$ and $95 \%$ air at $37^{\circ} \mathrm{C}$.

Hypoxia/reoxygenation (HR) model was induced by changing the medium to serum/glucose free DMEM medium and then incubating them in a Chamber (Wuxi PuHe Biological Medicine Technology Co., Ltd., Wuxi, China), which was flushed with $94 \%$ N2 and $5 \%$ CO2. After $4 \mathrm{~h}$ of hypoxia, the medium was replaced with fresh oxygenated medium containing serum and glucose, and the cells were returned to normoxic conditions for $4 \mathrm{~h}$. Hypoxic postconditioning (HPostC) was achieved by three cycles of $5 \mathrm{~min}$ of hypoxia and reoxygenation.

To explore the role of PHLPP1 on Akt and Mst1 phosphorylation as well as on cardiomyocyte injury, adenoviral vectors encoding siRNA against PHLPP1 $(\mathrm{MOI}, 50)$ were transfected into $\mathrm{H} 9 \mathrm{c} 2$ cells three days prior to hypoxia stimulation. The specific oligoribonucleotides for inhibiting PHLPP1 synthesis were 5'CUACCCAGUUCCAAAUUAUTT-3' and 5'-AUAAUUUGGAACUGGGUAGTT -3'. The negative control was 5'UUCUCCGAACGUGUCACGUTT-3' and 5'-ACGUGACACGUUCGGAGA ATT-3' (Gene Pharma, Shanghai, China). The specific PI3K/Akt inhibitor LY294002 (MCE, USA, $10 \mu \mathrm{M}$ ) or Mst1 inhibitor XMU-MP-1 (MCE, USA, $5 \mu \mathrm{M}$ ) was added to the H9c2 cardiomyoblasts medium before hypoxia stimulation [10, 21]. The cells incubated in normal glucose (5.5 mM) were divided into the following groups: 1) Control; 2) HR; 3) $H R+A d-s i P H L$; 4) HR+Ad-siPHL+LY; 5) HR+XMU. Meanwhile, to investigate the effect of high glucose on HPostC cardioprotection, the following experiments were performed:1) Control; 2) HR; 3) HR+HPostC; 4) high glucose (HG) $(25 \mathrm{mM}) ; 5) \mathrm{HG}+\mathrm{HR} ; 6) \mathrm{HG}+\mathrm{HR}+\mathrm{HPostC}$. The subgroups of H9c2 cells under high glucose were infected with adenoviral vectors encoding siRNA against PHLPP1. After transfection with 
Ad-siPHL or Ad-vector, cells were subjected to HR with or without HPostC as described above. An additional group of $\mathrm{H} 9 \mathrm{c} 2$ cells transfected with Ad-siPHL were treated with LY294002 before hypoxia stimulation.

\subsection{Determination of risk and infarct size}

At the end of the reperfusion, myocardial infarct size (IS) was assessed by $2 \%$ Evans blue and $2 \%$ TTC staining. The infarct size was calculated as the infarct area divided by area at risk (IS/AAR) as described previously [20].

\subsection{Measurement of creatinine kinase-MB (CK-MB)}

Serum creatine kinase-MB (CK-MB) level, a major indicator of myocardial injury, was measured by enzyme-linked immunoassay (Uscn Life Science Inc., China) as previously described [20].

\subsection{Detection of myocardial apoptosis}

Terminal-deoxynucleoitidyl transferase mediated nick end labeling (TUNEL) staining was used to assess cardiomyocyte apoptosis with an in situ cell detection kit (Roche Diagnostics, Germany) as we described [20]. The TUNEL-positive cells were counted under a high-power field (magnification, $\times 400$ ) and three randomly selected fields per heart were analyzed.

\subsection{Measurement of oxidative stress}

Reactive oxygen species (ROS) was measured by dihydroethidium (DHE) staining (Keygen Biotech, Co Ltd, Nanjing, China) according to the assay kit protocol. Cardiac frozen sections or H9C2 cells were incubated with DHE solution at $37^{\circ} \mathrm{C}$ for $30 \mathrm{~min}$ in a dark incubator. Then, the slides were visualized under fluorescence microscopy (magnification, $\times 400$ ). Three randomly fields were analyzed to calculate the mean data. The fluorescent intensity of DHE was analyzed with ImageJ software.

Levels of malondialdehyde (MDA) were measured with commercial assay kits (Nanjing Jiancheng Bioengineering Institute, Nanjing, China) as reported [20].

\subsection{Measurement of cell viability}

Cell viability was measured by the Cell Counting Kit-8 (MCE, USA) according to the manufacturer's instructions.

\subsection{Mitochondrial Membrane Potential Detection}

The mitochondrial membrane potential (MMP) in $\mathrm{H} 9 \mathrm{c} 2$ cardiomyoblasts was detected using the JC- 1 dye (MCE, USA). JC-1 was added to the cells after treatments and incubated in the dark for 20 min at $37^{\circ} \mathrm{C}$. $\mathrm{H} 9 \mathrm{c} 2$ cells was washed by PBS buffer for three times and subsequently examined under fluorescence microscopy (magnification, $\times 400$ ) at $530 \mathrm{~nm}$ (monomer form of JC-1, green) and at $590 \mathrm{~nm}$ (aggregate form of JC-1, red). Three randomly fields were analyzed. The fluorescent intensity was measured by ImageJ software. 


\subsection{Western blot analysis}

Western blot analysis was performed as previously reported [20]. Membranes were incubated overnight at $4^{\circ} \mathrm{C}$ with the following antibodies: phospho-Akt (Ser473) and phospho-Mst1(Thr183) (CST, USA) 1:1000, Akt and Mst1 (Abcam, UK) 1:1000, PHLPP1 (Proteintech, China) 1:500, Cleaved-Caspase3 (Affinity Biosciences, USA) 1:500. Then, the protein bands were incubated with a secondary antibody and visualized using an ECL kit.

\subsection{Statistical analysis}

All the values were expressed as mean \pm SD. One-way analysis of variance was performed to detect significant differences between the experimental groups (Graphpad Prism, San Diego, CA), followed by Newman-Keuls test for multiple comparisons. $P$ value $<0.05$ was considered statistically significant.

\section{Results}

\subsection{General characterization of animals}

As shown in Table 1, compared with the nondiabetic mice, diabetic animals manifested significantly increased water intake, food consumption and blood glucose $(P<0.05)$, while body weights were lower in diabetic mice than those in nondiabetic controls $(P<0.05)$.

Table 1

General characteristics

\begin{tabular}{|c|c|c|c|c|c|c|}
\hline Parameters & $\begin{array}{l}\text { Sham } \\
(n=10)\end{array}$ & $\begin{array}{l}\text { IR } \\
(n=17)\end{array}$ & $\begin{array}{l}\text { IR+IPostC } \\
(n=17)\end{array}$ & $\begin{array}{l}\text { DM } \\
(n=10)\end{array}$ & $\begin{array}{l}D M+I R \\
(n=17)\end{array}$ & $\begin{array}{l}\text { DM+IR+IPostC } \\
(n=17)\end{array}$ \\
\hline $\begin{array}{l}\text { Water intake } \\
(\mathrm{ml} / \mathrm{kg} / \text { day })\end{array}$ & $6.6 \pm 1.2$ & $\begin{array}{l}7.4 \pm \\
1.2\end{array}$ & $6.3 \pm 1.5$ & $\begin{array}{l}23.0 \pm \\
4.0^{*}\end{array}$ & $\begin{array}{l}28.8 \pm \\
6.8^{*}\end{array}$ & $29.7 \pm 7.0^{\star}$ \\
\hline $\begin{array}{l}\text { Food consumption } \\
\text { (g/kg/day) }\end{array}$ & $\begin{array}{l}4.4 \pm \\
0.7\end{array}$ & $\begin{array}{l}5.0 \pm \\
0.9\end{array}$ & $4.4 \pm 0.6$ & $\begin{array}{l}6.2 \pm \\
0.6^{*}\end{array}$ & $\begin{array}{l}6.7 \pm \\
1.0^{*}\end{array}$ & $6.9 \pm 1.2^{*}$ \\
\hline Body weight (g) & $\begin{array}{l}26.4 \pm \\
1.1\end{array}$ & $\begin{array}{l}26.2 \pm \\
1.7\end{array}$ & $\begin{array}{l}26.7 \pm \\
1.5\end{array}$ & $\begin{array}{l}23.0 \pm \\
1.1^{*}\end{array}$ & $\begin{array}{l}23.4 \pm \\
0.9^{*}\end{array}$ & $23.9 \pm 0.8^{\star}$ \\
\hline Plasma glucose (mM) & $\begin{array}{l}6.0 \pm \\
1.1\end{array}$ & $\begin{array}{l}6.3 \pm \\
0.7\end{array}$ & $5.9 \pm 1.0$ & $\begin{array}{l}24.3 \pm \\
3.4^{*}\end{array}$ & $\begin{array}{l}25.1 \pm \\
4.0^{*}\end{array}$ & $25.0 \pm 3.6^{*}$ \\
\hline \multicolumn{7}{|c|}{$\begin{array}{l}\text { All values are expressed as mean } \pm \text { SD. Water intake and food consumption were the average value of } \\
8 \text { weeks. Body weight and plasma glucose were measured among groups before inducing myocardial } \\
\text { ischemia/reperfusion. }{ }^{*} P<0.05 \text { vs their corresponding IR groups. }\end{array}$} \\
\hline
\end{tabular}




\subsection{Effects of IPostC on myocardial IR injury in mice}

As shown in Figures $1 \mathrm{a}$ and $1 \mathrm{~b}$, IPostC greatly reduced the infarct size in nondiabetic mice but failed to elicit similar effects in diabetic mice, and the infarct size in diabetic mice was larger than that in the corresponding control mice $(P<0.05)$. Nevertheless, AAR/LV did not differ significantly among all groups (Figure 1c).

CK-MB is a cardiac marker released from damaged cardiomyocytes after IR injury. As shown in Figure 1d, the plasma levels of CK-MB were elevated after myocardial IR injury $(P<0.05)$. IPostC significantly decreased the plasma CK-MB level in nondiabetic mice after IR injury $(P<0.05)$. Diabetes increased the post-ischemic CK-MB level in the $\mathrm{DM}+\mathrm{IR}$ and $\mathrm{DM}+\mathrm{IR}+\mathrm{IPostC}$ group compared with the IR group $(P<0.05)$. Meanwhile, the effect of IPostC in decreasing CK-MB release was blunted in the diabetic mice (DM+IR+IPostC group vs. DM+IR group, $P>0.05)$.

We evaluated the effect of IPostC on cardiomyocyte apoptosis after IR injury by TUNEL staining and cleaved Caspase3 expression. As shown in Figures 1e-1h, myocardial IR elevated the apoptotic index including the TUNEL-positive cells and the level of cleaved Caspase3 as compared with the corresponding sham group $(P<0.05)$. IPostC attenuated the cardiomyocyte apoptosis and the level of cleaved Caspase3 when compared with the IR group in nondiabetic mice $(P<0.05)$. However, the antiapoptotic effect of IPostC was abolished in the diabetic mice (DM+IR+IPostC group vs. DM+IR group, $P>0.05)$. Meanwhile, diabetic mice displayed increased cardiomyocyte apoptosis, as evidenced by the increased number of TUNEL-positive cells and the elevated level of cleaved Caspase 3 when compared with nondiabetic groups (DM+IR and DM+IR+IPostC groups vs IR group, $P<0.05$ ).

\subsection{Effects of IPostC on oxidative stress indicators in mice}

To investigate the antioxidant effects of IPostC, heart tissues were stained with DHE as a fluorescent marker of ROS and plasma MDA as one of the oxidative stress-related molecules was detected. As shown in Figures 2a-2c, myocardial IR significantly elevated the levels of ROS and MDA $(P<0.05)$. IPostC attenuated the levels of ROS and MDA in the IR+IPostC group when compared with the IR group $(P<0.05)$. However, the effect of IPostC in reducing superoxide anion generation was blunted in the diabetic mice (P>0.05). Meanwhile, the levels of ROS and MDA were elevated in the diabetic mice when compared with that in the nondiabetic mice $(P<0.05)$.

\subsection{Effects of IPostC on the levels of PHLPP1, Akt and Mst1 in mice}

As shown in Figure 3, the levels of PHLPP1 expression and Mst1 phosphorylation were decreased in the IR+IPostC group compared with the IR group $(P<0.05)$ while the level of Akt phosphorylation was increased $(P<0.05)$; however, these alternations were not observed in diabetic mice. The levels of PHLPP1 expression and Mst1 phosphorylation in diabetic heart were significantly higher than that in the 
corresponding nondiabetic mice after IR injury $(P<0.05)$, but diabetes remarkably reduced the phosphorylation level of Akt after myocardial IR (DM+IR and DM+IR+IPostC groups vs IR group, $P<0.05)$.

\subsection{Effects of PHLPP1/Akt/Mst1 signalling on the cellular injury in H9c2 Cardiomyocytes}

As shown in Figure 4a, western blot analysis showed that the expression of PHLPP1 was dramatically increased after $4 \mathrm{~h}$ reoxygenation compared with the baseline $(P<0.05)$. However, the expression of PHLPP1 decreased after $6 \mathrm{~h}$ reoxygenation compared with $4 \mathrm{~h}$ reoxygenation $(P<0.05)$.

As shown in Figure 4b, PHLPP1 knockdown significantly attenuated the cellular injury induced by HR compared with the HR group in normal glucose condition $(P<0.05)$. Moreover, the effect of PHLPP1 knockdown in reducing post-hypoxic cellular injury was partly abolished by LY294002 $(P<0.05)$. Meanwhile, the Mst1 kinase inhibitor XMU-MP-1 also reduced the post-hypoxic cellular injury $(P<0.05)$.

The bands of proteins in each group on western blot were shown in Figure 4c. The levels of PHLPP1 expression as well as Akt and Mst1 phosphorylation were increased in $\mathrm{H} 9 \mathrm{c} 2$ cardiomyocytes subjected to HR injury as compared to the Control group (Figures $4 d-4 f, P<0.05$ ). Compared with HR group, the level of PHLPP1 expression in the HR+Ad-siPHL and HR+Ad-siPHL+LY groups was obviously decreased (Figure $4 d, P<0.05)$. The level of Akt phosphorylation was significantly increased in the HR+Ad-siPHL group compared with the HR group (Figure 4e, P<0.05), but the increased level of Akt phosphorylation was blocked by LY294002. There was no significant difference in the levels of PHLPP1 and Akt phosphorylation between the HR group and HR+XMU group. The level of Mst1 phosphorylation was greatly decreased in the HR+Ad-siPHL, HR+Ad-siPHL+LY and HR+XMU groups compared with the HR group (Figure 4f, $P<0.05$ ).

\subsection{Effects of IPostC on the cellular injury induced in $\mathrm{H} 9 \mathrm{c} 2$ Cardiomyocytes}

As shown in Figure 5a, HPostC significantly elevated cell viability in the normal glucose condition $(P<0.05)$, but not in the high glucose condition. Meanwhile, $\mathrm{H} 9 \mathrm{c} 2$ cardiomyocytes exposed to high glucose showed reduced cell viability when compared with cells exposed to normal glucose after HR injury $(P<0.05)$.

The levels of MDA release and ROS production were increased in H9c2 cardiomyocytes exposed to high glucose as compared to the HR group (Figures $5 b, 5 c$ and $5 e, P<0.5$ ). The posthypoxic elevations of MDA and ROS generation in $\mathrm{H} 9 \mathrm{c} 2$ cells were reduced by HPostC when cells were exposed to normal glucose $(P<0.5)$ but not to high glucose.

The mitochondrial permeability transition pore (MPTP), which resulted in the change of MMP, has been proposed to be a critical determinant of myocardial injury. JC-1 is a fluorescent lipophilic carbocyanine dye used to measure MMP. HR stimulation significantly increased the JC-1 ratio (green/red) and cleaved Caspase3 expression, and HPostC reversed these changes in the normal glucose condition (Figures $5 \mathrm{~d}$ 
and $5 f-5 h, P<0.5)$, but not in the high glucose condition. Meanwhile, the posthypoxic JC-1 ratio (green/red) and cleaved Caspase3 expression were elevated in $\mathrm{H} 9 \mathrm{c} 2$ cardiomyocytes exposed to high glucose as compared to the HR group $(P<0.5)$.

\subsection{Effects of IPostC on the levels of PHLPP1, Akt and Mst1 in H9c2 Cardiomyocytes}

As shown in Figure 6, HPostC greatly increased the level of Akt phosphorylation and decreased the levels of PHLPP1 expression and Mst1 phosphorylation in the normal glucose condition $(P<0.05)$, but not in the high glucose condition. High glucose obviously elevated the levels of PHLPP1 expression and Mst1 phosphorylation and reduced the level of Akt phosphorylation compared with the HR group $(P<0.05)$.

\subsection{Effects of HPostC with PHLPP1 knockdown on cellular injury in $\mathrm{H} 9 \mathrm{c} 2$ cells exposed to high glucose}

Both $\mathrm{HG}+\mathrm{Ad}$-siPHL+HR and $\mathrm{HG}+\mathrm{Ad}-\mathrm{siPHL}+\mathrm{HR}+\mathrm{HPostC}$ groups significantly increased cell viability and decreased cleaved Caspase3 expression in $\mathrm{H} 9 \mathrm{c} 2$ cardiomyocytes exposed to high glucose when compared with $\mathrm{HG}+$ Ad-vector+HR group (Figures $7 \mathrm{a}-\mathrm{c}, \mathrm{P}<0.5$ ), but these changes were reversed by LY294002 $(P<0.5)$.

PHLPP1 gene knockdown significantly attenuated the levels of posthypoxic MDA (Figure 7(d)) and ROS production (Figures 7e and 7f) in H9c2 cardiomyocytes exposed to high glucose (HG+Ad-siPHL+HR or $\mathrm{HG}+\mathrm{Ad}$-siPHL+HR+HPostC group vs HG+Ad-vector+HR, $P<0.05)$, and these effects were blocked by LY294002 $(P<0.5)$.

\subsection{Effects of HPostC with PHLPP1 knockdown on the levels of PHLPP1, Akt and Mst1 in H9c2 cells exposed to high glucose}

The bands of proteins in each group on western blot were shown in Figure 8a. In Figure 8b, the expression of PHLPP1 was significantly inhibited in $\mathrm{H} 9 \mathrm{c} 2$ cardiomyocytes transfected by adenoviral vectors encoding siRNA against PHLPP1 compared with baseline $(P<0.5)$, and there were no significant differences between the HG+Ad-vetor+HR group and HG+Ad-vector+HR+HPostC group. The level of Akt phosphorylation was elevated in $\mathrm{HG}+\mathrm{Ad}$-siPHL+HR and $\mathrm{HG}+\mathrm{Ad}$-siPHL+HR+HPostC groups when compared with $\mathrm{HG}+$ Ad-vector+HR group (Figure 8c, $\mathrm{P}<0.05$ ), but the enhanced level of Akt phosphorylation was blocked by LY294002 $(P<0.5)$. PHLPP1 knockdown markedly decreased the expression of phosphorylated Mst1 compared with the corresponding groups treated with adenoviral vectors encoding scramble siRNA (Figure $8 d, P<0.05$ ). 


\section{Discussion}

In the current study, we have demonstrated that IPostC or HPostC can attenuate postischemic injury reflected by reduced infarct size, cell viability, plasma CK-MB, ROS generation and cellular apoptosis in nondiabetic mice or in $\mathrm{H} 9 \mathrm{c} 2$ cells exposed to normal glucose. Moreover, we found that the cardioprotective effect induced by IPostC or HPostC is associated with the attenuation of PHLPP1 expression to mediate not only through the activation of Akt but also through the inactivation of Mst1. Meanwhile, we showed that inhibition of PHLPP1 or Mst1 can decrease the cellular damage in in vitro, but the effect is blocked by LY294002. Importantly, we showed that impaired IPostC cardioprotection in the diabetic heart is associated with excessive expression of PHLPP1. PHLPP1 knockdown preserves the protective effect induced by HPostC in $\mathrm{H} 9 \mathrm{c} 2$ cells exposed to high glucose not only through the activation of Akt but also through the inactivation of Mst1.

IPostC has been proposed as a strategy against myocardial IR injury, and the cardioprotective effect has been confirmed in animal studies [22]. Our previous study also confirmed that remote limb ischemic postconditioning could protect against myocardial IR injury in mice [4]. In this study, a cardiac IR injury mouse model was established. AAR/LV ratio did not differ among all groups, indicating that the model in our study is reliable. Our data demonstrated that IPostC obviously attenuated the myocardial infarct size, plasma CK-MB release, cardiomyocytes apoptosis and oxidative stress after $45 \mathrm{~min}$ of ischemia and $2 \mathrm{~h}$ of reperfusion in mice. Meanwhile, HPostC significantly decreased the cellular damage, cleaved Caspase3 expression, oxidative stress and mPTP opening level reflected by JC-1 ratio (green/red) in H9c2 cells exposed to normal glucose after $4 \mathrm{~h}$ of hypoxia and $4 \mathrm{~h}$ of reoxygenation. Taken together, IPostC has a potent protective effect against cardiac IR injury.

Akt is an important protective kinase in the heart injury. It is well known that activation of Akt kinase protects the heart against IR injury [23]. PHLPP1 is an endogenous negative regulator of Akt signaling in the heart. PHLPP1 knockdown elevates the Akt activity in cardiomyocytes and, in turn, provides protection against IR injury [12]. Mst1 is the second important PHLPP1 target [14]. Mst1 is a vital kinase of the Hippo signaling pathway that regulates the development and homeostasis of heart by mediating cell proliferation, apoptosis and cell fate decisions [24]. The data demonstrated that HR stimulation gradually elevated PHLPP1 expression from 2 to $6 \mathrm{~h}$, with the peak increase in PHLPP1 expression occurring after 4 $h$ of reoxygenation. This result suggests that PHLPP1 is involved in the pathological process of myocardial HR injury. Meanwhile, to investigate the role of PHLPP1 in cardiomyocyte death, we examined whether PHLPP1 knockdown alone is sufficient to attenuate cellular apoptosis. The data indicated that siRNA-mediated knockdown of PHLPP1 gene could mitigate the cardiomyocyte damage induced by HR under normal glucose condition, accompanied with increased Akt activity and decreased Mst1 activity. However, the protective effect of PHLPP1 knockdown was blocked by PI3K/Akt inhibitor LY294002. Meanwhile, we found that Mst1 inhibitor XMU-MP-1 could also reduce HR-mediated cardiomyocyte injury. After myocardial IR or HR injury, the levels of PHLPP1 expression as well as Akt and Mst1 phosphorylation were elevated in the cardiomyocytes. Moreover, IPostC or HPostC could greatly attenuate the PHLPP1 expression in the cardiomyocyte, concomitant with the significant increment of Akt 
phosphorylation and reduction of Mst1 phosphorylation. Therefore, the data indicated that downregulation of PHLPP1 expression plays an important role in the effect of IPostC cardioprotection not only through the activation of Akt but also through the inactivation of Mst1.

Ischemic postconditioning results in a significant reduction in infarct size in young, healthy animals. However, major cardiovascular comorbidities such as hyperlipidemia, diabetes, and aging interfere with these cardioprotective mechanisms, thereby limiting the protective effects of myocardial ischemic postconditioning [25]. It is tempting to see the effect of comorbidities on the failure of ischemic postconditioning in clinical translation. Therefore, such studies would be necessary to identify important pathways of cardioprotection that could be inactive in the presence of major cardiovascular comorbidities. Our data indicated that the myocardial infarct size, apoptosis and cellular injury were markedly increased in diabetic mice or $\mathrm{H} 9 \mathrm{c} 2$ cells exposed to high glucose, and the result was consistent with our previous studies $[9,10,20]$. In addition, we observed that hyperglycemia eliminated the cardiac protective effects of IPostC, which fits well with previously reported studies that hyperglycemia could abolish the benefits of ischemic postconditioning [26,27]. Previous studies have shown that increased cardiac PTEN and decreased cardiac TOPK or DJ-1 or STAT3 are responsible for the loss of diabetic heart sensitivity to ischemic or pharmacological postconditioning [10,26-28], but the underlying molecular mechanism is completely unclear. Meanwhile, some studies indicated that several agents such hydrogen sulfide, deferoxamine and $\mathrm{N}$-acetylcysteine were utilized to restore the myocardial protective effect of sevoflurane or ischemic postconditioning in diabetes [29-31], but most of them alone could protect heart against IR injury [32-34]. Therefore, the efficient and effective method has yet to be developed. have myocardial protection. In our study, our data indicated that either in STZ-induced diabetic mice or in H9c2 cells exposed to high glucose, the cardiac protective effects of IPostC were blocked, accompanied by increased PHLPP1 expression and Mst1 phosphorylation as well as decreased Akt phosphorylation. We have mentioned above that IPostC protected heart against IR injury via inhibition of PHLPP1 expression to elevate Akt phosphorylation and reduce Mst1 phosphorylation. Therefore, these data suggest that the underlying mechanism attributable to the limiting cardioprotection induced by IPostC is likely the abnormality of PHLPP1/Akt/Mst1 signaling pathway in diabetes. Then, we further confirmed these observations by inhibiting PHLPP1 expression in H9c2 cells exposed to high glucose. Our data showed that PHLPP1 knockdown by adenoviral vectors encoding siRNA decreased the cellular damage and oxidative stress in $\mathrm{H} 9 \mathrm{c} 2$ cells exposed to high glucose, accompanied by increased Akt phosphorylation and decreased Mst1 phosphorylation. Moreover, the protective effects induced by PHLPP1 knockdown were blocked by LY294002. IPostC was added in the PHLPP1 knockdown H9c2 cells exposed to high glucose to partly decrease the cellular injury and oxidative stress, but it was not statistically significant. Taken together, these findings indicated that hyperglycemia-induced overexpression of PHLPP1 played a key role in the lost effect of IPostC cardioprotection.

\section{Conclusions}

In conclusion, our findings indicate that IPostC protects against myocardial IR injury possibly through the PHLPP1/Akt/Mst1 signaling pathway, while the excessive expression of PHLPP1 in the hyperglycemic 
condition contributes to the abolishment of cardioprotective effects by IPostC. In addition, PHLPP1 knockdown preserves the effect of IPostC cardioprotection not only through the activation of Akt but also through the inactivation of Mst1. Therefore, downregulation of PHLPP1 could be considered as a novel alternative to attenuate the reperfusion injury in diabetic patients with ischemic heart disease.

\section{Declarations}

\section{Data Availability}

The data used to support the findings of this study are included within the article.

\section{Conflicts of Interest}

The authors declare that there is no conflict of interest regarding the publication of this article.

\section{Authors' contributions}

SG, HS, YQ and YM conceived and designed the experiments; $Y Q, Y M, Y J$ and $X L$ performed the experiments; $Y Q, Y M, Y J, X L, S G$ and $H S$ analyzed the data; $Y Q, Y M, H Z, S G, L D, T W$ and HS contributed to discussion and wrote the paper. All authors read and approved the final paper.

\section{Funding}

This work was supported by grants from the National Natural Science Foundation of China (NO. 81800721), the Natural Science Foundation of Huai'an (HAB201802), the Post-doctoral Foundation of China and Jiangsu (2020M671387, 2020Z393).

\section{Ethics approval}

The care of animals and all animal experiments were implemented after being reviewed and approved by the Institutional Animal Use and Care Committee at Nanjing Medical University.

\section{Consent to Participate}

Not applicable.

\section{Consent for Publication}

Not applicable.

\section{References}

1. Virani SS; Alonso A; Aparicio HJ et al. Heart disease and stroke statistics-2021 update: A report from the American Heart Association. Circulation. 2021;8(143):e254-e743. 
2. Frohlich GM; Meier P; White SK; Yellon DM; Hausenloy DJ. Myocardial reperfusion injury: looking beyond primary PCI. Eur Heart J. 2013;23(34):1714-1722.

3. Rosenberg JH; Werner JH; Moulton MJ; Agrawal DK. Current modalities and mechanisms underlying cardioprotection by ischemic conditioning. J Cardiovasc Transl Res. 2018;4(11):292-307.

4. Gao S; Zhan L; Yang Z et al. Remote limb ischaemic postconditioning protects against myocardial ischaemia/reperfusion injury in mice: activation of JAK/STAT3-mediated Nrf2-antioxidant signalling. Cell Physiol Biochem. 2017;3(43):1140-1151.

5. Tyagi S; Singh N; Virdi JK; Jaggi AS. Diabetes abolish cardioprotective effects of remote ischemic conditioning: evidences and possible mechanisms. J Physiol Biochem. 2019;1(75):19-28.

6. Hausenloy DJ; Yellon DM. New directions for protecting the heart against ischaemia-reperfusion injury: targeting the Reperfusion Injury Salvage Kinase (RISK)-pathway. Cardiovasc Res. 2004;3(61):448-460.

7. Tsang A; Hausenloy DJ; Mocanu MM; Yellon DM. Postconditioning: a form of "modified reperfusion" protects the myocardium by activating the phosphatidylinositol 3-kinase-Akt pathway. Circ Res. 2004;3(95):230-232.

8. Penna C; Andreadou l; Aragno M et al. Effect of hyperglycaemia and diabetes on acute myocardial ischaemia-reperfusion injury and cardioprotection by ischaemic conditioning protocols. $\mathrm{Br} \mathrm{J}$ Pharmacol. 2020;23(177):5312-5335.

9. Qiu Y; Wu Y; Meng M et al. GYY4137 protects against myocardial ischemia/reperfusion injury via activation of the PHLPP-1/Akt/Nrf2 signaling pathway in diabetic mice. J Surg Res. 2018;225:29-39.

10. Gao S; Wang R; Dong S et al. Inactivation of TOPK caused by hyperglycemia blocks diabetic heart sensitivity to sevoflurane postconditioning by impairing the PTEN/PI3K/Akt signaling. Oxid Med Cell Longev. 2021;2021:6657529.

11. Gao T; Furnari F; Newton AC. PHLPP: a phosphatase that directly dephosphorylates Akt, promotes apoptosis, and suppresses tumor growth. Mol Cell. 2005;1(18):13-24.

12. Miyamoto S; Purcell NH; Smith JM et al. PHLPP-1 negatively regulates Akt activity and survival in the heart. Circ Res. 2010;4(107):476-484.

13. Ling P; Lu TJ; Yuan CJ; Lai MD. Biosignaling of mammalian Ste20-related kinases. Cell Signal. 2008;7(20):1237-1247.

14. Qiao M; Wang Y; Xu X et al. Mst1 is an interacting protein that mediates PHLPPs' induced apoptosis. Mol Cell. 2010;4(38):512-523.

15. Nakamura M; Zhai P; Del RD; Maejima Y; Sadoshima J. Mst1-mediated phosphorylation of Bcl-xL is required for myocardial reperfusion injury. JCI Insight. 2016;5(1):e86217.

16. Wu W; Ziemann M; Huynh K et al. Activation of Hippo signaling pathway mediates mitochondria dysfunction and dilated cardiomyopathy in mice. Theranostics. 2021;18(11):8993-9008.

17. Odashima M; Usui S; Takagi H et al. Inhibition of endogenous Mst1 prevents apoptosis and cardiac dysfunction without affecting cardiac hypertrophy after myocardial infarction. Circ Res. 
2007;9(100):1344-1352.

18. Zhang M; Zhang L; Hu J et al. Mst1 coordinately regulates autophagy and apoptosis in diabetic cardiomyopathy in mice. Diabetologia. 2016;11(59):2435-2447.

19. Feng $X$; Wang $S$; Yang $X$ et al. Mst1 knockout alleviates mitochondrial fission and mitigates left ventricular remodeling in the development of diabetic cardiomyopathy. Front Cell Dev Biol. 2020;8:628842.

20. Gao S; Yang Z; Shi R et al. Diabetes blocks the cardioprotective effects of sevoflurane postconditioning by impairing Nrf2/Brg1/HO-1 signaling. Eur J Pharmacol. 2016;779:111-121.

21. Triastuti E; Nugroho AB; Zi M et al. Pharmacological inhibition of Hippo pathway, with the novel kinase inhibitor XMU-MP-1, protects the heart against adverse effects during pressure overload. $\mathrm{Br} \mathrm{J}$ Pharmacol. 2019;20(176):3956-3971.

22. Heusch G; Rassaf T. Time to give up on cardioprotection? A critical appraisal of clinical studies on ischemic pre-, post-, and remote conditioning. Circ Res. 2016;5(119):676-695.

23. Fujio Y; Nguyen T; Wencker D; Kitsis RN; Walsh K. Akt promotes survival of cardiomyocytes in vitro and protects against ischemia-reperfusion injury in mouse heart. Circulation. 2000;6(101):660-667.

24. Mia MM; Singh MK. The Hippo signaling pathway in cardiac development and diseases. Front Cell Dev Biol. 2019;7:211.

25. Varga ZV; Giricz Z; Bencsik P et al. Functional genomics of cardioprotection by ischemic conditioning and the influence of comorbid conditions: implications in target identification. Curr Drug Targets. 2015;8(16):904-911.

26. Li H; Yao W; Liu Z et al. Hyperglycemia abrogates ischemic postconditioning cardioprotection by impairing AdipoR1/Caveolin-3/STAT3 signaling in diabetic rats. Diabetes. 2016;4(65):942-955.

27. Liu M; Zhou B; Xia ZY et al. Hyperglycemia-induced inhibition of DJ-1 expression compromised the effectiveness of ischemic postconditioning cardioprotection in rats. Oxid Med Cell Longev. 2013;2013:564902.

28. Xue R; Lei S; Xia ZY et al. Selective inhibition of PTEN preserves ischaemic post-conditioning cardioprotection in STZ-induced Type 1 diabetic rats: role of the PI3K/Akt and JAK2/STAT3 pathways. Clin Sci (Lond). 2016;5(130):377-392.

29. Zhang J; Cai X; Zhang Q et al. Hydrogen sulfide restores sevoflurane postconditioning mediated cardioprotection in diabetic rats: Role of SIRT1/Nrf2 signaling-modulated mitochondrial dysfunction and oxidative stress. J Cell Physiol. 2021;7(236):5052-5068.

30. Xie P; Yang L; Talaiti A et al. Deferoxamine-activated hypoxia-inducible factor-1 restores cardioprotective effects of sevoflurane postconditioning in diabetic rats. Acta Physiol (Oxf). 2017;2(221):98-114.

31. Lin J; Wang T; Li Y et al. N-Acetylcysteine restores sevoflurane postconditioning cardioprotection against myocardial ischemia-reperfusion injury in diabetic rats. J Diabetes Res. 2016;2016:9213034. 
32. Calvert JW; Jha S; Gundewar S et al. Hydrogen sulfide mediates cardioprotection through Nrf2 signaling. Circ Res. 2009;4(105):365-374.

33. Reddy BR; Kloner RA; Przyklenk K. Early treatment with deferoxamine limits myocardial ischemic/reperfusion injury. Free Radic Biol Med. 1989;1(7):45-52.

34. Pasupathy S; Tavella R; Grover S et al. Early use of N-acetylcysteine with nitrate therapy in patients undergoing primary percutaneous coronary intervention for ST-Segment-Elevation myocardial infarction reduces myocardial infarct size (the NACIAM Trial [N-acetylcysteine in acute myocardial infarction]). Circulation. 2017;10(136):894-903.

\section{Figures}

\section{Figure 1}

Effects of IPostC on myocardial IR injury in mice. (a) Representative images of TTC-stained hearts. Infarct size (IS: white); area at risk (AAR: red and white); perfused area (blue). (b) Comparison of area of infarct size normalized to the area at risk (IS/AAR). (c) Comparison of area at risk per left ventricle (AAR/LV). (d) Plasma CK-MB. (e) TUNEL staining. (DAPI: nuclei, blue; TUNEL: apoptosis nuclei, green; magnification, $\times 400)$. (f) Quantification of TUNEL-positive cardiomyocytes (\% of total). (g) Representative western blot bands. (h) Quantitative data of cleaved Caspase3. All values are presented as mean \pm S.D. ( $\mathrm{n}$ $=5$ or 7 per group). ${ }^{*} P<0.05$ compared with IR group; ${ }^{\# ~} P<0.05$ compared with $D M+I R$ group.

\section{Figure 2}

Effects of IPostC on the oxidative stress indicators in mice. (a) Images of dihydroethidium (DHE) staining (magnification, $\times 400)$. (b) Quantitative analysis of DHE expression. (c) Plasma malondialdehyde (MDA). All values are presented as mean \pm S.D. $\left(\mathrm{n}=5\right.$ per group). ${ }^{*} P<0.05$ compared with IR group; ${ }^{\#} P<0.05$ compared with DM+IR group.

\section{Figure 3}

Effects of IPostC on the levels of PHLPP1, Akt and Mst1 in mice. (a) Representation western blot bands. Protein expression of PHLPP1 (b), Akt (c), and Mst1 (d) and their phosphorylation status. All values are presented as mean \pm S.D. ( $n=5$ per group). ${ }^{*} P<0.05$ compared with IR group; ${ }^{\#} P<0.05$ compared with DM+IR group. 


\section{Figure 4}

Effects of PHLPP1/Akt/Mst1 signalling on the cellular injury in H9c2 Cardiomyocytes. (a) Representative western blot of PHLPP1 compared with GADPH. (b) Cell viability. (c) Representation western blot bands. Protein expression of PHLPP1 (d), Akt (e), and Mst1 (f) and their phosphorylation status. All values are presented as mean \pm S.D. ( $\mathrm{n}=3-5$ per group). ${ }^{*} P<0.05$ compared with HR group; ${ }^{\#} P<0.05$ compared with different time point of hypoxia/reoxygenation.

\section{Figure 5}

Effects of IPostC on the cellular injury in H9c2 Cardiomyocytes. (a) Cell viability. (b) Serum level of MDA. (c) Representative images of DHE staining (magnification, $\times 400$ ). (d) Representative images of JC-1 staining (magnification, $\times 400$ ). (e) Quantitative analysis of DHE expression. (f) Quantitative analysis of JC-1 ratios (green/red). (g) Representative western blot bands. (h) Quantitative data of cleaved Caspase3. All values are presented as mean \pm S.D. ( $\mathrm{n}=3-5$ per group). ${ }^{*} P<0.05$ compared with HR group; ${ }^{\#} P<0.05$ compared with $\mathrm{HG}+\mathrm{HR}$ group.

\section{Figure 6}

Effects of IPostC on the levels of PHLPP1, Akt and Mst1 in H9c2 Cardiomyocytes. (a) Representation western blot bands. Protein expression of PHLPP1 (b), Akt (c), and Mst1 (d) and their phosphorylation status. All values are presented as mean \pm S.D. ( $n=3$ per group). ${ }^{*} P<0.05$ compared with HR group; \# $P<0.05$ compared with $\mathrm{HG}+\mathrm{HR}$ group.

\section{Figure 7}

Effects of HPostC with PHLPP1 knockdown on the cellular injury in $\mathrm{H} 9 \mathrm{c} 2$ cells exposed to high glucose. (a) Cell viability. (b) Representative western blot bands. (c) Quantitative data of cleaved Caspase3. (d) Serum level of MDA. (e) Representative images of DHE staining (magnification, $\times 400$ ). (f) Quantitative analysis of DHE expression. All values are presented as mean \pm S.D. ( $n=3-5$ per group). ${ }^{\star} P<0.05$ compared with $\mathrm{HG}+\mathrm{Ad}$-vector+HR group; ${ }^{\#} P<0.05$ compared with $\mathrm{HG}+\mathrm{Ad}-\mathrm{vector}+\mathrm{HR}+\mathrm{HPostC}$ group.

\section{Figure 8}


Effects of HPostC with PHLPP1 knockdown on the levels of PHLPP1, Akt and Mst1 in H9c2 cells exposed to high glucose. (a) Representation western blot bands. Protein expression of PHLPP1 (b), Akt (c), and Mst1 (d) and their phosphorylation status. All values are presented as mean \pm S.D. ( $n=3$ per group). * $P<0.05$ compared with HG+Ad-vector+HR group; ${ }^{\#} P<0.05$ compared with HG+Ad-vector+HR+ HPostC group 